\title{
AVALIAÇÃO DA CINÉTICA E EQUILÍBRIO DA SECAGEM DO BAGAÇO DE CANA-DE-AÇÚCAR PARA APLICAÇÃO COMO ADSORVENTE
}

\author{
F.B. SCHEUFELE ${ }^{1 *}$, A. DIÓRIO ${ }^{1}$, A.N. MÓDENES ${ }^{2}$, R. ERGAMASCO ${ }^{1}$, N.C. PEREIRA ${ }^{1}$ \\ ${ }^{1}$ Universidade Estadual de Maringá, Departamento de Engenharia Química \\ ${ }^{2}$ Universidade Estadual do Oeste do Paraná, Centro de Engenharias e Ciências Exatas \\ *E-mail para contato: fabianoscheufele@gmail.com
}

\begin{abstract}
RESUMO - Este trabalho teve como objetivo a avaliação da cinética e equilíbrio do processo de secagem do bagaço com a finalidade de melhorar a sua eficiência num processo de remoção do corante têxtil. Os experimentos de cinética foram realizados em estufa com circulação de ar, em camada fina. $\mathrm{O}$ equilíbrio de sorção de umidade foi determinado pelo método estático, entre 35 e $65^{\circ} \mathrm{C}$. Para todas as temperaturas avaliadas, o processo de secagem apresentou período de taxa de secagem decrescente, o qual foi melhor representado pelo modelo Dois Termos. Os valores de difusividade variaram entre $6,47.10^{-9}$ e $3,97.10^{-8} \mathrm{~m}^{2} \mathrm{~s}^{-1}$ e a energia de ativação foi $30,20 \mathrm{~kJ} \mathrm{~mol}^{-1}$. Os dados de equilíbrio apresentaram dependência da temperatura, com isotermas no formato sigmoidal melhor representados pelo modelo de Oswin. Em todas as temperaturas avaliadas, o processo de secagem apresentou cinética favorável, viabilizando sua utilização como adsorvente.
\end{abstract}

\section{INTRODUÇÃO}

A indústria têxtil se caracteriza pelo elevado consumo de água e pela produção de grandes quantidades de efluentes contendo altas concentrações de corantes, sua liberação sem o tratamento é nociva aos sistemas aquáticos. Dentre os tratamento empregados aos efluentes têxteis a adsorção se destaca devido à alta capacidade de remoção destes poluentes. No entanto, em algumas situações seu custo pode inviabilizar o processo, principalmente, devido aos adsorventes tradicionalmente utilizados como o carvão ativado, por exemplo. Diversas pesquisas tem sido realizadas com o objetivo de encontrar materiais alternativos, dentre eles destacam-se os materiais naturais, resíduos agrícolas e industriais (Módenes et al. 2013).

O bagaço de cana-de-açúcar apresenta-se como um resíduo agroindustrial do setor sucroalcooleiro. Atualmente, sua destinação está relacionada com a queima e produção de energia nas destilarias, no entanto ainda existe um excedente o qual acarreta problemas de armazenamento (Pandey et al. 2000; Cardona et al. 2010). Devido à abundância e de elevada disponibilidade, justifica-se a utilização deste material em diversas aplicações, dentre elas o uso como material adsorvente.

Devido à elevada umidade do bagaço de cana faz-se necessário reduzir a umidade por um processo de secagem para viabilizar seu emprego, bem como realizar o seu armazenamento. A redução da atividade de água, evita a proliferação de micro-organismos que possam vir a degradar ou alterar as características do material (Ghazanfari et al. 2006; Castell-Palou \& Simal, 2011). Além disso, no caso do emprego como material adsorvente é fundamental a retirada da umidade, pois as moléculas de água ligadas à superfície podem prejudicar o processo sortivo. Torna-se necessário, portanto, realizar-se o estudo do processo de secagem com o 
intuito de verificar se as condições de secagem do material influenciam na sua aplicação como adsorvente.

Desta forma, os objetivos deste trabalho foram investigar o efeito da temperatura sobre a cinética e o equilíbrio de secagem do bagaço de cana-de-açúcar, realizar a modelagem da cinética de secagem em camada fina, ajustar as isotermas de sorção aos dados experimentais de equilíbrio e, ainda, determinar o coeficiente de difusividade efetiva e a energia de ativação deste resíduo.

\section{MATERIAIS E MÉTODOS}

\subsection{Materiais}

O bagaço de cana-de-açúcar utilizado nos ensaios experimentais foi coletado na Usina Santa Terezinha - USAÇÚCAR, unidade de Tapejara, Paraná - Brasil. O material foi acondicionando em sacos plásticos e armazenado em congelador na temperatura de $-15^{\circ} \mathrm{C}$.

\subsection{Métodos}

A avaliação da cinética de secagem foi realizada em estufa com circulação de ar em camada fina, em triplicata. A massa inicial do bagaço de cana utilizado foi de 3,0 g, a qual foi distribuída uniformemente sobre uma placa plana, mantendo-se uma altura do leito menor que $8 \mathrm{~mm}$. As amostras submetidas ao processo de secagem foram pesadas a cada $1 \mathrm{~min}$ em balança analítica até atingir peso constante. As temperaturas de secagem do bagaço avaliadas variaram de 30 a $80^{\circ} \mathrm{C} \pm 1^{\circ} \mathrm{C}$, sendo que o teor de umidade (b.s.) foi calculado pela Equação (1).

$X(b . s)=.\frac{m_{\text {úmida }}-m_{\text {seca }}}{m_{\text {seca }}}$

em que $X$ (b.s) é o teor de umidade da amostra em base seca $\left(\mathrm{g} \mathrm{H}_{2} \mathrm{O} \mathrm{g}^{-1}\right.$ sólido seco), $m_{\text {úmida }}$ é a massa da amostra (g) e $m_{\text {seca }}$ é a massa seca obtida em estufa $105^{\circ} \mathrm{C}$ por $24 \mathrm{~h}(\mathrm{~g})$.

Na Tabela 1 são apresentados os modelos de secagem em camada fina empregados para a descrição dos dados experimentais. Dentre os modelos em camada fina empregados aos dados de cinética de secagem existem os difusivos e os empíricos. O transporte de umidade no material depende de parâmetros como difusividade, condutividade térmica e os coeficientes de transferência de massa (internos e externos), entretanto, em algumas situações a literatura utiliza o termo constante de secagem $(k)$ como a combinação destes parâmetros de transporte (Geankoplis, 1993). A parâmetro constante de secagem ( $k$ ) é mais adequado para o design e otimização do processo, pois requer menos esforço matemático, ao contrário das equações diferenciais parciais clássicas de transferência de calor e massa (Mujumdar, 2006).

Tabela 1 - Modelos cinéticos de secagem em camada fina.

\begin{tabular}{lll}
\hline Nome & Modelo cinético & $(2)$ \\
\hline Lewis & $X_{a d}=\exp (-k t)$ \\
Page & $X_{a d}=\exp \left(-k t^{n}\right)$ \\
Midilli-Kucuk & $X_{a d}=a \exp \left(-k t^{n}\right)+b t$ \\
Dois termos & $X_{a d}=a \exp \left(-k_{1} \mathrm{t}\right)+b \exp \left(-k_{2} \mathrm{t}\right)$ & $(3)$ \\
\hline
\end{tabular}


em que $X_{a d}$ é o teor de umidade adimensionalizado (-), $X$ é o teor de umidade em um dado instante de tempo, $X_{o}$ é o teor de umidade inicial da amostra e $X_{e}$ é o teor de umidade de equilíbrio. As constantes $k, n, a, b, k_{1} e k_{2}$ são parâmetros ajustáveis dos modelos, e $t$ é o tempo de secagem ( $\min )$.

\subsection{Difusividade Efetiva e Energia de Ativação}

O processo de secagem de materiais de alta umidade é complexo, envolvendo transferência simultânea de calor e massa (Ghazanfari et al. 2006; Castell-Palou \& Simal, 2011), no entanto em determinados materiais a secagem pode ser limitada pela difusão no interior da partícula. Nestas situações a equação da difusão de Fick, dada pela Equação (6), pode descrever o processo, na qual a difusividade efetiva $\left(D_{\text {eff }}\right)$ é um parâmetro que representa o termo condutivo de todos os mecanismos de transferência de massa.

$\frac{\partial X}{\partial t}=D_{e f f}\left(\nabla^{2} X\right)$

Uma solução analítica desta equação diferencial da difusão da umidade para placa plana, foi proposta por CRANK (1975), através de uma expansão em séries. A solução baseia-se nas considerações em que o mecanismo de transferência de massa que limita o processo é a difusão da umidade no interior da partícula, a umidade é uniforme na amostra e que o coeficiente de difusividade é constante, e independente da umidade da amostra. Entretanto, para longos tempos de secagem, é possível considerar $\mathrm{n}=1$, de forma que a solução fica resumida apenas ao primeiro termo do somatório, conforme a Equação (7).

$X_{a d}=\frac{X-X_{e}}{X_{o}-X_{e}}=\frac{8}{\pi^{2}} \exp \left(\frac{\pi^{2} D_{e f f} t}{4 L^{2}}\right)$

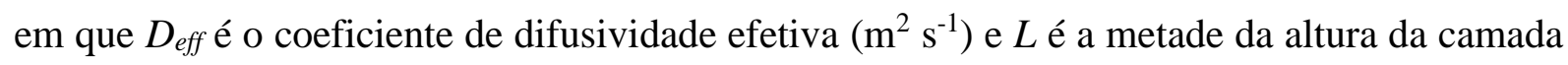
do material $(\mathrm{m})$.

\subsection{Isotermas de sorção}

As isotermas de equilíbrio de umidade do bagaço de cana foram obtidas pelo método gravimétrico estático nas temperaturas de 35,50 e $65^{\circ} \mathrm{C}\left( \pm 1^{\circ} \mathrm{C}\right)$. Foram preparadas soluções salinas saturadas $\left(\mathrm{CH}_{3} \mathrm{CO}_{2} \mathrm{~K}, \mathrm{~K}_{2} \mathrm{CO}_{3}, \mathrm{KCl}, \mathrm{MgCl}_{2} .6 \mathrm{H}_{2} \mathrm{O}, \mathrm{Mg}\left(\mathrm{NO}_{3}\right) 2, \mathrm{NaNO}_{2} \mathrm{e} \mathrm{NaCl}\right)$, as quais mantém uma atmosfera de umidade controlada que varia de acordo com o sal e a temperatura do ensaio. Os valores da atividade de água $\left(a_{w}\right)$ para cada uma das condições são apresentados por BAHLOUL et al. (2008).

O aparato experimental consistiu de um frasco de vidro vedado e um pedestal, no qual adicionou-se a amostra de bagaço de cana-de-açúcar. Os experimentos foram realizados em triplicata, sendo que inicialmente inseriu-se $30 \mathrm{~mL}$ de solução salina nos frascos, levando-os à estufa na temperatura desejada do experimento por $24 \mathrm{~h}$ para estabilização da temperatura. $\mathrm{Na}$ sequência pesou-se $0,1 \mathrm{~g}$ do bagaço, previamente seco em estufa, o qual foi disposto sobre o pedestal. Os aparatos foram colocados em estufa na temperatura de cada ensaio $\left(35,50\right.$ e $\left.65^{\circ} \mathrm{C}\right)$, as amostras foram pesadas em balança analítica $( \pm 0,0001 \mathrm{~g})$ a cada $48 \mathrm{~h}$ até atingir o equilíbrio. O conteúdo da massa seca (b.s.) das amostras foi obtido por gravimetria, levando-as em estufa 
a $105^{\circ} \mathrm{C}$ por $24 \mathrm{~h}$. A umidade de equilíbrio foi calculada pela Equação (1), sendo que a massa úmida corresponde à massa obtida no equilíbrio do experimento de sorção.

Um grande número de equações têm sido propostas na literatura para a predição da relação entre $X_{\mathrm{e}}, a_{w}$ e $T$ (Mujumdar, 2006). Aos dados experimentais de sorção de umidade do bagaço de cana-de-açúcar, nas temperaturas 35,50 e $65^{\circ} \mathrm{C}$, foram ajustados as equações matemáticas apresentadas na Tabela 2.

Tabela 2 - Isotermas de sorção empregados nos dados de equilíbrio do bagaço de cana.

\begin{tabular}{cc}
\hline Modelo & Equação \\
\hline Oswin (1946) & $X_{e}=A\left(\frac{a_{w}}{1-a_{w}}\right)^{B}$ \\
Henderson (1952) & $X_{e}=A\left[\frac{1}{T} \ln \left(\frac{1}{1-a_{w}}\right)\right]^{B}$ \\
Halsey (1976) & $X_{e}=A\left[T \ln \left(\frac{1}{a_{w}}\right)\right]^{-B}$ \\
BET (1938) & $X_{e}=\frac{X_{m} C a_{w}}{\left(1-a_{w}\right)\left(1+C a_{w}-a_{w}\right)}$ \\
\hline
\end{tabular}

Em que $T$ é a temperatura do ensaio $\left({ }^{\circ} \mathrm{C}\right), A, B, C$ são os parâmetros ajustáveis e $X m$ é o conteúdo de umidade na monocamada molecular em (b.s.) ( $\left.\mathrm{g} \mathrm{g}^{-1}\right)$. A estimativa dos parâmetros dos modelos foi realizada por meio de regressões não lineares (método de Levenberg-Marquardt) utilizando o software Origin $8^{\circledR}$, sendo que melhor ajuste apresentado pelos modelos foi verificado avaliando-se o coeficiente de determinação do modelo $\left(R^{2}\right)$ e o menor qui-quadrado reduzido $\left(\chi^{2}\right)$.

\section{RESULTADOS E DISCUSSÃO}

\subsection{Cinética de secagem}

Os resultados de cinética de secagem do bagaço de cana-de-açúcar em camada fina são apresentados na Figura 1(a), na qual verifica-se que a umidade inicial do bagaço foi de aproximadamente $0,20 \mathrm{~g}$ de água por g de sólido seco, possuindo uma elevada capacidade de sorção de água tornando-o portanto um material altamente hidroscópico. Observa-se ainda que o aumento da temperatura de secagem influencia a velocidade da secagem do material, acelerando o processo. A umidade de equilíbrio também é afetada pela temperatura de secagem, alcançando-se menores valores nas temperaturas superiores. A cinética de secagem do bagaço de cana é favorável, pois mesmo em baixas temperaturas curtos tempos de equilíbrio foram obtidos, em $30^{\circ} \mathrm{C}$, por exemplo, a umidade estabilizou-se em torno de $60 \mathrm{~min}$ de secagem.

$\mathrm{Na}$ Figura 1(b) encontram-se os resultados de taxa de secagem para a faixa de temperatura avaliada. Os resultados sugerem que maiores temperaturas levam a taxas de secagem mais acentuadas, além disso, em todas as temperaturas avaliadas o material apresentou período de taxa decrescente de secagem. O período de taxa decrescente de secagem é um indicativo que a difusão de umidade do interior da partícula do material até a superfície limita o processo global de secagem, sendo característico de sólidos não porosos (e.g. madeira, papel, amido e fibras têxteis) (Geankoplis, 1993). O bagaço de cana de açúcar possui uma estrutura muito similar à estes materiais, sendo composto, basicamente, por polímeros de alto peso molecular. 

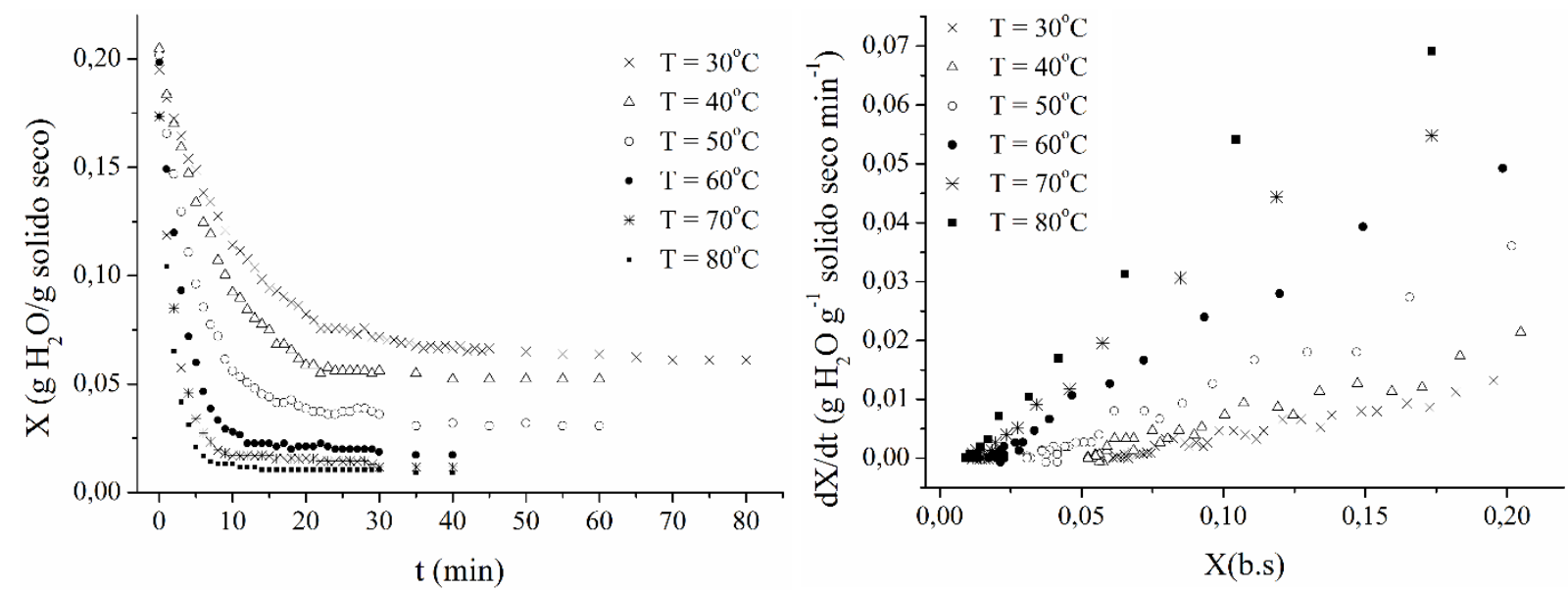

Figura 1 - Curvas de secagem (a) e curvas de taxa de secagem (b) do bagaço de cana-deaçúcar para as temperaturas de 30 a $80^{\circ} \mathrm{C}$.

Na modelagem cinética da secagem do bagaço de cana-de-açúcar, dentre os modelos cinéticos em camada fina (Tabela 1), o modelo que melhor representou os dados experimentais para todas as temperaturas avaliadas foi o modelo Dois Termos $\left(\overline{R^{2}}=0,9980 e \overline{\chi^{2}}=1,33 \cdot 10^{-4}\right)$. Entretanto, o modelo difusivo de Lewis apresentou resultados similares $\left(\overline{R^{2}}=0,9962\right.$ e $\overline{\chi^{2}}=$ $2,49.10^{-4}$ ), tornando-se mais conveniente avaliá-lo devido a sua simplicidade e por ser um modelo teórico, o qual é aplicável aos dados experimentais obtidos, visto que o processo de difusão de umidade no sólido controla o processo global de secagem.

Além dos modelos em camada fina, avaliou-se a cinética de secagem do bagaço através dos valores do coeficiente de difusividade. Na Tabela 3 são apresentados os valores da constante de taxa de secagem $(k)$, estimada pelo modelo de Lewis, juntamente com os valores de $D_{\text {eff }}$ obtidos pela solução analítica de Crank. Nota-se que em todas temperaturas ambos modelos se ajustaram aos dados, apresentando valores de $R^{2}$ maiores que 0,993. Assim, é possível afirmar que os modelos descrevem o processo de secagem em camada fina do bagaço adequadamente, na faixa de temperatura avaliada. Observa-se ainda que os parâmetros constante de secagem $(k)$ e difusividade efetiva $\left(D_{\text {eff }}\right)$ são dependentes da temperatura. Seus valores aumentam nas temperaturas mais elevadas, indicando que os mecanismos de transferência de massa ocorrem mais rapidamente, acelerando o processo de secagem.

Tabela 3. Parâmetros estimados do modelo cinético de Lewis e valores de difusividade obtidos pelo modelo de Crank.

\begin{tabular}{c|cc|cc}
\hline $\mathbf{T}\left({ }^{\mathbf{0}} \mathbf{C}\right)$ & $\boldsymbol{k}\left(\mathbf{s}^{\mathbf{- 1}}\right)$ & $\boldsymbol{R}^{\mathbf{2}}$ & $\boldsymbol{D}_{\text {eff }}\left(\mathbf{m}^{\mathbf{2}} \mathbf{s}^{-1}\right)$ & $\mathbf{R}^{\mathbf{2}}$ \\
\hline $\mathbf{3 0}$ & 0,07814 & 0,99824 & $6,45.10^{-9}$ & 0,99692 \\
$\mathbf{4 0}$ & 0,12985 & 0,99684 & $1,09.10^{-8}$ & 0,99526 \\
$\mathbf{5 0}$ & 0,18332 & 0,99384 & $1,39.10^{-8}$ & 0,99568 \\
$\mathbf{6 0}$ & 0,29416 & 0,99652 & $2,43.10^{-8}$ & 0,99614 \\
$\mathbf{7 0}$ & 0,39659 & 0,99345 & $2,98.10^{-8}$ & 0,99437 \\
$\mathbf{8 0}$ & 0,53118 & 0,99807 & $3,97.10^{-8}$ & 0,99390 \\
\hline
\end{tabular}

A expressão de Arrhenius foi capaz de representar os resultados de coeficiente de difusividade em função da temperatura. $\mathrm{O}$ ajuste aos dados experimentais do bagaço de canade-açúcar apresentou um coeficiente de determinação $R^{2}$ de 0,984 e um qui-quadrado reduzido de $2,63 \cdot 10^{-18}$. A constante de difusividade $D_{0}$ foi estimada em $1,18 \cdot 10^{-3} \mathrm{~m}^{2} \mathrm{~s}^{-1}$ e a energia de ativação $\left(E_{a}\right)$ obtida foi de $30,200 \mathrm{~kJ} \mathrm{~mol}^{-1}$. 


\subsection{Isotermas de sorção}

Os resultados de equilíbrio de sorção de água no bagaço de cana-de-açúcar, obtidos nas temperaturas de 35 e 50 e $65^{\circ} \mathrm{C}$ são apresentados na Figura 2 (a), na qual é possível observar que os dados experimentais apresentaram um comportamento de umidade de equilíbrio nãolinear crescente em função da atividade de água, configurando um formato sigmoidal. A temperatura exerce influência na umidade de equilíbrio, de forma que nas temperaturas superiores atingiu-se menores umidades de equilíbrio no material, assim como obtido na cinética de secagem. Por exemplo, na temperatura de $35^{\circ} \mathrm{C}$ a maior umidade de equilíbrio obtida foi em torno de $0,12 \mathrm{~g}$ de $\mathrm{H}_{2} \mathrm{O} / \mathrm{g}$ de sólido seco, enquanto na temperatura de $65^{\circ} \mathrm{C}$ este valor decaiu para aproximadamente $0,05 \mathrm{~g}$ de $\mathrm{H}_{2} \mathrm{O} / \mathrm{g}$ de sólido seco.

Segundo Bahloul et al. (2008), a umidade de equilíbrio diminui com o acréscimo da temperatura, assumindo um mesmo valor de $a_{w}$, devido ao estado de excitação das moléculas de água. Em temperaturas elevadas as moléculas encontram-se em estados de excitação elevados aumentando a distância entre si e levando à diminuição, portanto, da sua força atrativa. Este fenômeno leva à uma redução da quantidade de água sorvida, para uma dada umidade relativa, em função do aumento da temperatura.

Aos dados experimentais de equilíbrio de sorção de água no bagaço de cana-de-açúcar, foram ajustados as isotermas (Tabela 2), para cada uma das temperaturas avaliadas. Os ajustes das isotermas aos dados experimentais podem ser visualizados na Figura 2.
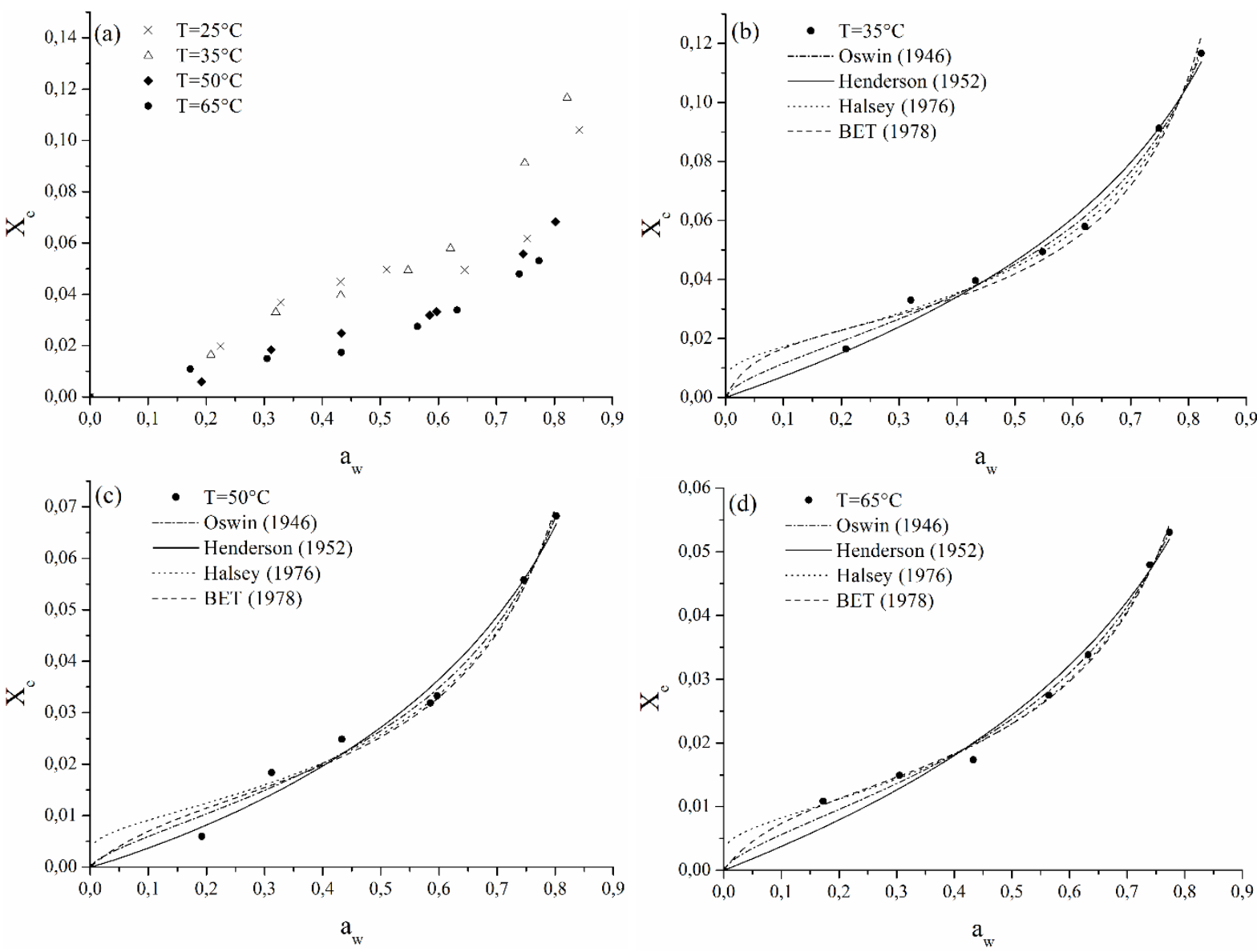

Figura 2 - (a) Dados de equilíbrio de sorção de água no bagaço de cana-de-açúcar; Ajuste das isotermas de sorção nas temperaturas de: (b) $35^{\circ} \mathrm{C}$, (c) $50^{\circ} \mathrm{C}$ e (d) $65^{\circ} \mathrm{C}$. 
Nas Figuras 2 (b-d) observa-se que os modelos de isotermas empregados ajustaram-se aos dados experimentais de equilíbrio para o bagaço em todas as temperaturas avaliadas. As curvas apresentam um formato sigmoidal, formando por três regiões. A primeira região compreendida, aproximadamente, entre $0<a_{w}<0,30$ representa a região onde se formam camadas monomoleculares, ou seja, nesta região a água fica retida na superfície do material, fortemente ligada à sítios individuais, não encontrando-se disponível para reações. A segunda região, entre $0,3<a_{w}<0,5$, corresponde à uma transição, na qual formam-se duplas e múltiplas camadas, neste caso ligadas mais fracamente. Por fim, a última região $\left(0,5<a_{w}<1,0\right)$ corresponde às situações em que ocorre a condensação capilar preenchendo totalmente o material com água. Nesta situação a água é mais facilmente removida, caso as condições de temperatura e UR sejam favoráveis (Bahloul et al. 2008; Geankoplis, 1993; Mujumdar, 2006).

Este comportamento é característico de produtos higroscópicos, sendo comumente observada em produtos agrícolas. As isotermas de sorção de umidade da maioria dos produtos alimentícios e fibrosos são não-lineares, e geralmente, apresentam formato sigmoidal, sendo classificados como isotermas do tipo II, conforme a classificação de Brunauer (Ruthven, 1984).

No que se refere à aplicação do bagaço de cana como material adsorvente, caso a umidade de equilíbrio atingida após sua secagem seja elevada, estas moléculas de água fortemente ligadas podem interferir na capacidade de adsorção do material, pois poderão ocupar os sítios ativos impedindo a ligação da molécula de corante à superfície.

Os resultados da estimativa dos parâmetros obtidos pelas regressões não lineares das isotermas de sorção de água do bagaço de cana-de-açúcar para as diferentes temperaturas avaliadas são apresentadas na Tabela 4.

Tabela 4. Parâmetros das isotermas ajustadas aos dados de sorção do bagaço de cana.

\begin{tabular}{ccccc}
\hline Modelo & Parâmetro & $\mathbf{T}=\mathbf{3 5}^{\circ} \mathbf{C}$ & $\mathbf{T}=\mathbf{5 0}^{\circ} \mathbf{C}$ & $\mathbf{T}=\mathbf{6 5}^{\circ} \mathbf{C}$ \\
\hline & $X_{m}$ & 0,02205 & 0,01447 & 0,01267 \\
BET (1938) & $C$ & 19,10258 & 6,89355 & 9,82657 \\
& $\chi^{2}$ & $2,31.10^{-5}$ & $8,74.10^{-6}$ & $1,74.10^{-6}$ \\
& $R^{2}$ & 0,98422 & 0,98382 & 0,99487 \\
\hline \multirow{3}{*}{ Oswin (1946) } & $A$ & 0,04513 & 0,02649 & 0,02375 \\
& $B$ & 0,62254 & 0,67827 & 0,65619 \\
& $\chi^{2}$ & $8,96.10^{-6}$ & $6,24.10^{-6}$ & $2,39.10^{-6}$ \\
& $R^{2}$ & 0,99387 & 0,98846 & 0,99298 \\
\hline \multirow{2}{*}{ Henderson (1952) } & $A$ & 2,23041 & 2,5188 & 2,21510 \\
& $B$ & 0,98882 & 1,05883 & 0,99279 \\
& $\chi^{2}$ & $1,94.10^{-5}$ & $8,38.10^{-6}$ & $5,76.10^{-6}$ \\
& $R^{2}$ & 0,98672 & 0,9845 & 0,98308 \\
\hline & $A$ & 0,53880 & 0,55647 & 0,60544 \\
& $B$ & 0,78500 & 0,86639 & 0,85840 \\
& $\chi^{2}$ & $1,29.10^{-5}$ & $9,16.10^{-6}$ & $1,37.10^{-6}$ \\
& $R^{2}$ & 0,99114 & 0,98306 & 0,99596 \\
\hline
\end{tabular}

Através dos valores de $R^{2}$ e $\chi^{2}$ apresentados na Tabela 4 observa-se que as isotermas de sorção avaliadas se ajustaram aos dados experimentais nas três temperaturas estudadas, apresentando-se valores de $R^{2}$ próximos à unidade, e baixos valores de qui-quadrado reduzido. $\mathrm{O}$ modelo de Oswin obteve o melhor ajuste nas temperaturas de $35^{\circ} \mathrm{C}$ e $50^{\circ} \mathrm{C}$, enquanto na temperatura de $65^{\circ} \mathrm{C}$ o modelo de Halsey melhor representou os dados experimentais. É 
possível visualizar na Figura 2, entretanto, que o modelo de Halsey não se ajusta adequadamente nos valores de $a_{w}$ inferiores à 0,1 .

\section{CONCLUSÕES}

O estudo da cinética e equilíbrio de secagem do bagaço de cana-de-açúcar mostrou que o material é altamente higroscópico, apresentando elevados teores de umidade. Desta forma, verifica-se que este bagaço proveniente da indústria necessita ser submetido à secagem para evitar sua deterioração e na aplicação em diferentes fins, como por exemplo no caso da adsorção. A avaliação cinética mostrou que o processo é favorável e rápido, mesmo em baixas temperaturas de secagem. O material apresentou período decrescente de secagem, o qual indica que a difusão de umidade no interior da partícula controla o processo. Foi possível descrever a cinética de secagem tanto pelos modelos em camada fina, quanto pela solução analítica da Lei de Fick proposta por Crank. Através da constante de secagem $(k)$ e do coeficiente de difusividade $\left(D_{\text {eff }}\right)$ nota-se que a velocidade do processo é fortemente dependente da temperatura de secagem. Os resultados de equilíbrio de sorção de água também indicaram uma dependência da umidade de equilíbrio com a temperatura. Em todas as temperaturas avaliadas observou-se um perfil sigmoidal, característico de produtos higroscópicos alimentícios e fibrosos. Dentre as isotermas de sorção avaliadas o modelo de Oswin melhor representou os dados. A avaliação da cinética de secagem e equilíbrio de sorção fornecem informações fundamentais para a aplicação do bagaço de cana-de-açúcar como material adsorvente, pois verifica-se que as moléculas de água podem estar fortemente ligadas impedindo o processo de adsorção do corante. Desta forma, conclui-se que o processo de secagem é essencial para possibilitar o uso deste material como adsorvente.

\section{REFERÊNCIAS}

BAHLOUL, N.; BOUDHRIOUA, N.; KECHAOU, N. Moisture desorption-adsorption isotherms and isosteric heats of sorption of Tunisian olive leaves (Olea europaea L.). Ind. crop. prod., v.28, p.162176, 2008.

CARDONA, C.A.; QUINTERO, J.A.; PAZ, I.C. Production of bioethanol from sugarcane bagasse: Status and perspectives, Bioresour. Technol., v.101, p.4754-4766, 2010.

CASTELL-PALOU A.; SIMAL S. Heat pump drying kinetics of a pressed type cheese, LWT - Food Sci. Technol., v.44, p.489-494, 2011.

GEANKOPLIS, C. J. Transport Processes and Unit Operations. Prentice-Hall, 3 Ed., 921 p., 1993.

GHAZANFARI A.; EMAMI S.; TABIL L.G.; PANIGRAHI S. Thin-Layer Drying of Flax Fiber: II. Modeling Drying Process Using Semi-Theoretical and Empirical Models, Drying Technol., v.24, p.1637-1642, 2006.

MÓDENES, A.N.; ROSS, A.A.; SOUZA, B.V.; DOTTO, J.; GERALDI, C.Q.; ESPINOZAQUIÑONES, F.R.; KROUMOV, A.D. Biosorption of BF-4B Reactive Red Dye by using Leaves of Macrophytes Eichhornia crassipes. Int. J. Bioautomation, v.17(1), p.33-44, 2013.

MUJUMDAR, A. S. Handbook of industrial drying. 3 ed., CRC Press, Taylor \& Francis Group, 2006.

PANDEY A.; SOCCOL C.R.; NIGAM P.; SOCCOL V.T. Biotechnological potential of agro-industrial residues. I. Sugarcane bagasse. Bioresource Technology, v.74(2), p.69-80, 2000.

RUTHVEN, D. M. Principles of adsorption and adsorption process, John Wiley \& Sons, New York, 432 p., 1984. 